Original Research Paper

\title{
Endotoxin Activity Assay: An Experience of a Southern-Italy Hospital
}

\author{
${ }^{1}$ Linda Degl'Innocenti, ${ }^{2}$ Flaviano Di Paola, ${ }^{2}$ Rizzo Marianna and ${ }^{1}$ Gerardino Amato \\ ${ }^{1}$ Laboratory of Clinical Pathology and Microbiology, A.O.R.N. “Antonio Cardarelli”, Naples, Italy \\ ${ }^{2}$ Department of Diagnostic Pathology and Laboratory, \\ Division of Laboratory Medicine, Istituto Nazionale Tumori "Fondazione G. Pascale"-IRCCS, Naples, Italy
}

Article history

Received: 2014-10-03

Revised: 2014-10-07

Accepted: 2014-12-29

Corresponding Author:

Flaviano Di Paola,

Department of Diagnostic

Pathology and Laboratory,

Division of Laboratory Medicine,

Istituto Nazionale Tumori

"Fondazione G. Pascale"-IRCCS,

Naples, Italy

Email: f.dipaola@istitutotumori.na.it

\begin{abstract}
Endotoxin Activity Assay is a novel and rapid test capable of differentiating Gram-negative sepsis from infections carried by other bacteria. The test, moreover, represents a useful tool in the management of the septic patient. We show the data collected during a 6-year period experience, related to samples from septic patients admitted in ICU; for each patient we performed an EAA test and a blood culture with an automated system. Our aim was to evaluate the effectiveness of the EAA test, alone or in combination with blood cultures, in ICU setting. Our experience confirms that the measure of the endotoxin levels, along with the execution of a traditional blood culture, provides a useful tool in the management of the critical patient. The high predictive negative value of the EAA test, moreover, allow to rule-out a Gram-negative bloodstream infection.
\end{abstract}

Keywords: EAA, Endotoxin, Sepsis, Gram-Negative Infections

\section{Introduction}

Sepsis is a severe clinical syndrome due to an impaired regulation of the immune system in reaction to the infectious diseases, which may rapidly evolve into the multiple failure of various organs and systems. The early detection of the different clinical signs could be difficult, due to the aspecific symptoms involved, leading to the poor prognosis of the patient (Carrigan et al., 2004). Sepsis is still nowadays a serious issue, because of its high rate of incidence and mortality. In facts, sepsis is worldwide a primary cause of morbidity and mortality, especially in patients with impaired immune system, patients in critical conditions and elderly in ICU. For example, there are about 750.000 cases of severe sepsis leading to 215.000 deaths per year in the US alone (Angus et al., 2001). The incidence in Europe appears to be similar (Alberti et al., 2002).

Currently, the gold standard for the diagnosis of sepsis is the blood culture and, due to the long turnaround times, an empirical broad-spectrum antibiotics therapy is often necessary. This approach, however, shows several disadvantages, such as the selection of drugresistant bacterial strains, with the subsequent increasing in mortality rate and in the costs related to the infectious diseases management.

It is currently known that there is a peculiar difference between Gram-positive and Gram-negative bacteria in triggering the sepsis pro-inflammatory cascade process. The Gram-positive ones, in facts, cause sepsis by producing and excreting specific exotoxins, as well as through cell-wall lipoteicoic acid action (Sriskandan et al., 1999); Gram-negative bacteria, on the other hand,trigger sepsis by the presence of the endotoxins, that is the membrane Lipopolysaccaride (LPS) present as a constituent of the Gram-negative cell wall (Ulevitch et al., 1999). In ICU setting, Gramnegative bacteria represent the most frequent etiological agents of bloodstream infections (Gaynes et al., 2005; Sievert et al., 2013), as also observed in our experience. On the basis of these considerations, also pushed by ICU divisions of our Hospital (A.O.R.N. "Antonio Cardarelli", Naples), has become urgent the need for a rapid quantification of the probability of sepsis by Gramnegative bacteria, measured in whole-blood samples in patients with clinically relevant signs. For this purpose, we show the data collected using the new FDA-approved technology Endotoxin Activity Assay (EAA) in patients with suspected septic shock.

The test is based on the reaction between endotoxin and a lipid-A specific monoclonal antibody, amplified by blood neutrophils, that in presence of Zymosan yields an oxidative reaction measured by the emission of chemiluminescence (due to the presence of luminol in the reaction mixture) proportional to the LPS-antibody (antigen-antibody) complex concentration (Romaschin et al., 1998). Wholeblood samples (at least $2 \mathrm{~mL}$ ) should be collected in EDTA-tubes, stored at room temperature and tested 
within $3 \mathrm{~h}$. The test rules-out a Gram-negative infection with values less than 0.40 EAA units, while results greater than 0.60 EAA units indicate a high risk of Gram-negative sepsis and/or septic shock.

We evaluated the endotoxin activity levels in septic or septic-suspected patients and, for each patient, blood culture samples were also drawn. Our aim was to establish a correlation between endotoxin levels and Gram-negative isolation from blood samples, in order to evaluate whether the endotoxin levels measurement in circulating peripheral blood could clinically impact patient's sepsis management or not.

\section{Material and Methods}

\section{Endotoxin Activity Assay}

We measured endotoxin activity levels in 650 samples, collected between March 2007 and December 2013 from patients admitted to the ICU of the A.O.R.N. "Antonio Cardarelli", Naples. All the whole-blood samples, sent to the Microbiology Division of our hospital, were tested using EAA (Estor s.p.a., Milan) within $3 \mathrm{~h}$ of receipt. The cut-off levels were used as suggested by the manufacturer (Table 1).

\section{Blood Cultures}

Blood culture samples for each patient, were also collected and tested using a Bactec system (BectonDickinson Italia, Milan) and following a 5-days negative protocol. The bottles flagged as positive by the system were subcultured on solid media, followed by identification and antimicrobial susceptibility testing of the isolated bacteria.

\section{Results}

We received 654 samples, collected from March 2007 to December 2013 both from our hospital and from other Institutes' ICUs. Four samples were excluded from the data collection because of the inability of performing the endotoxin activity assay. Two samples, in facts, were clotted and the other two were excessively diluted and probably contained interfering substances. We tested 650 samples and the results are shown in Table 2.

The results of the endotoxin activity levels showed 407 patients $(62 \%)$ with $\mathrm{EAA} \geq 0,60$ units (range 0.71 $1.02)$; in 152 patients (24\%) were found EAA levels between 0.40 and 0.59 units (range 0.48-0.58); in 91 samples $(14 \%)$ were found an EAA level $<0.40$ units (range 0.00-0.39). The results of the blood cultures from the same patients showed that in 404 of 407 patients with high endotoxin levels $(\geq 0.60$ EAA units) were found at least one Gram-negative positive blood culture. In the remaining patients, whose samples showed moderate or low EAA levels, no positive blood culture were drawn.

\section{Discussion}

EAA is an easy-performing assay, since it requires a single whole-blood sample collection, it is quick (with a less than $1 \mathrm{~h}$ turn-around time) and allows differentiating Gram-negative sepsis from infections in which such bacteria are to be excluded. In 404 of 407 positive samples we found at least one positive blood culture carried by Gram-negative bacteria, with the exception of 3 cases of false positive results, due to bowel perforation ( 2 cases) and intestinal infarction ( 1 case). Otherwise, in the remaining 152 intermediate and 91 negative samples, no positive blood culture were observed. As mentioned before, septic shock is one of the most critical clinical conditions in emergency medicine, often accompanied by poor prognosis, in which the rapidity of the diagnosis and intervention probably assumes the most important role. It is necessary, therefore, to introduce in the clinical routine new assays with which clinicians, along with blood cultures, could more accurately and more rapidly make sepsis diagnosis.

Table 1. EAA levels cut-offs

\begin{tabular}{ll}
\hline Endotoxin Activity levels (EAA units) & Interpretation \\
\hline $0.0-0.39$ & Gram-negative sepsis ruled-out \\
$0.40-0.59$ & Moderate risk for severe sepsis \\
$\geq 0.60$ & High risk for severe sepsis \\
\hline
\end{tabular}

Table 2. Endotoxin activity levels of the patients

Blood cultures

\begin{tabular}{llll}
\hline & Blood cultures & & \\
EAA levels (units) & N. patients (\%) & N. positive (\%) & N. negative (\%) \\
\hline $0.0-0.39$ & $91(14 \%)$ & $0(0 \%)$ & $91(100 \%)$ \\
$0.40-0.59$ & $152(24 \%)$ & $0(0 \%)$ & $52(100 \%)$ \\
$\geq 0.60$ & $407(62 \%)$ & $404(99 \%)$ & $3(1 \%)$ \\
\hline
\end{tabular}




\section{Conclusion}

The use of the blood culture in combination with endotoxin activity assay provides the best predictiveness and rapidity in microbiological diagnosis of sepsis. The use of EAA does not have to be considered as a replacement of the traditional tests (e.g., blood cultures), still necessary in order to retrieve information about antimicrobial susceptibility patterns or further investigations, but represents a supplemental test that allows to minimize the time interval between the blood collection and the completion of cultural investigations. Moreover, has been observed that the endotoxin activity assay, in spite of having a low specificity (about 44\%), has a high predictive negative value which makes this test very effective in ruling-out Gram-negative sepsis.

\section{Acknowledgement}

The authors declare that there is no conflict of interests regarding the publication of this article.

\section{Author's Contributions}

All authors equally contributed in this study.

\section{Ethics}

This article is original and contains unpublished material. The corresponding author confirms that all of the other authors have read and approved the manuscript and no ethical issues involved.

\section{References}

Alberti, C., C. Brun-Buisson, H. Burchardi, C. Martin and S. Goodman et al., 2002. Epidemiology of sepsis and infection in ICU patients from an international multicentre cohort study. Int. Care Med., 28: 108121. PMID: 11907653

Angus, D.C., W.T. Linde-Zwirble, J. Lidicker, G. Clermont and J. Carcillo et al., 2001. Epidemiology of severe sepsis in the United States: analysis of incidence, outcome and associated costs of care. Crit. Care Med., 29: 1303-1310. PMID: 11445675

Carrigan, S.D., G. Scott and M. Tabrizian, 2004. Toward resolving the challenges of sepsis diagnosis. Clin. Chem., 50: 1301-1314. PMID: 15166107
Gaynes, R., J.R. Edwards and NNISS, 2005. Overview of nosocomial infections caused by gram-negative bacilli. Clin. Infect. Dis., 41: 848-854. DOI: $10.1086 / 432803$

Romaschin, A.D., D.M. Harris, M.B. Ribeiro, J. Paice and D.M. Foster et al., 1998. A rapid assay of endotoxin in whole blood using autologous neutrophil dependent chemiluminescence. J. Immunol. Meth., 212: 169-185. PMID: 9672205

Sievert, D.M., P. Ricks, J.R. Edwards, A. Schneider and J. Patel et al., 2013. Antimicrobial-resistant pathogens associated with healthcare-associated infections: Summary of data reported to the national healthcare safety network at the centers for disease control and prevention, 2009-2010. Infect. Cont. Hospital Epidemiol., 34: 1-14. PMID: 23221186

Sriskandan, S. and J. Cohen, 1999. Gram-positive sepsis. Mechanisms and differences from gram-negative sepsis. Infect. Dis. Clin. North Am., 13: 397-412. PMID: 10340174

Ulevitch, R.J. and P.S. Tobias, 1999. Recognition of gram-negative bacteria and endotoxin by the innate immune system. Current Opinion Immunol., 11: 1922. PMID: 10047547 